\title{
Another Rare Unstable Hemoglobinopathy: Hemoglobin Casper/Southampton Associated with Moyamoya Disease
}

\author{
Asim Haque $^{a}$ Douglas J. Quint ${ }^{b}$ Valerie P. Castle ${ }^{c}$ Steven M. Leber ${ }^{a, d}$ \\ ${ }^{a}$ Department of Neurology, ${ }^{b}$ Division of Neuroradiology, Department of Radiology, and \\ Divisions of ${ }^{\mathrm{C}} \mathrm{Hematology/Oncology}$ and ${ }^{\mathrm{d}}$ Neurology, Department of Pediatrics, University \\ of Michigan Hospitals, Ann Arbor, Mich., USA
}

\section{Introduction}

Strokes and their sequelae are a known complication of sickle cell disease [1], yet far less is known about the neurological complications of rarer hemoglobinopathies. In this report, a case of stroke and moyamoya disease is described in a child with hemoglobin ( $\mathrm{Hb}$ ) Casper, a rare and unstable hemoglobin mutation.

\section{Case Report}

A 9-year-old right-handed girl with a history of hemolytic anemia, status post-splenectomy, and previously identified right frontal encephalomalacia presented with a 1-month history of episodic right upper extremity weakness and 'garbled' speech.

Her neurological examination was notable for episodes of nonfluent speech and dysarthria, some right-left confusion, and episodic distal right arm weakness and paresthesias. Her laboratory studies were notable for a macrocytic hemolytic anemia and thrombocytosis, but negative for hypercoagulable, inflammatory, cardiac, infectious, and autoimmune markers. Extensive hematological testing performed for anemia 3 years earlier had revealed an $\mathrm{Hb}$ Casper mutation. However, she never returned for follow-up until her current presentation. There was no known family history of strokes, cardiac issues or hematologic abnormalities.

An initial MRI showed multiple foci of impeded diffusion in the left frontal lobe (fig. 1a, b). Fluid-attenuated inversion-recovery (FLAIR) imaging showed an increased T2 signal involving the left hemispheric leptomeninges and perivascular spaces (fig. 1c) and chronic right frontal encephalomalacia (not shown). Subsequent conventional angiography showed an occlusion of the petrous right internal carotid artery (ICA) with distal reconstitution of the right middle cerebral artery (MCA) and A1 segments through prominent vascular collaterals (not shown), and an occlusion of the proximal supraclinoid left ICA (fig. 1d arrow) with skull base collateral reconstitution of the left anterior cerebral artery (ACA) (fig. 1d, e). Left 


\section{Cerebrovascular
Diseases}
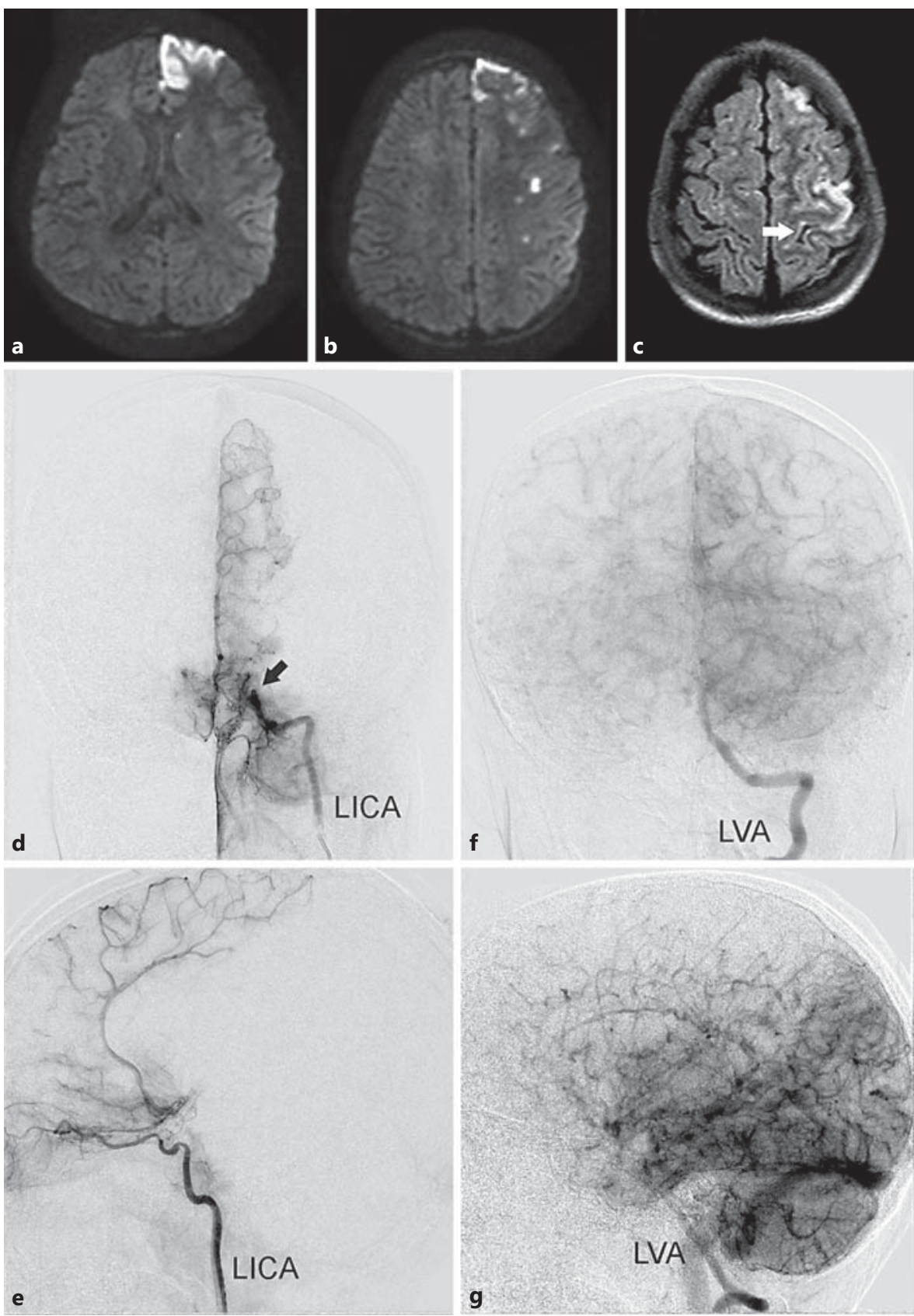

Fig. 1. MRI (a-c) obtained at the time of presentation and digital subtraction angiography $(\mathbf{d}-\mathbf{g})$ obtained several weeks later. Areas of impeded diffusion are noted on the axial diffusion weighted imaging $(\mathbf{a}, \mathbf{b})$ involving the left ACA and MCA territories. Axial FLAIR imaging (c) shows an increased T2 signal involving the leptomeninges and perivascular spaces, likely representing the 'ivy sign' (white arrow) of pial collateralization. Digital subtraction angiography of the left ICA ( $\mathbf{d}$ - anteroposterior, $\mathbf{e}$ - lateral) shows the abrupt termination of the proximal supraclinoid left ICA (black arrow) with reconstitution of the left ACA branches through the skull base (predominantly ophthalmic) collateral vasculature consistent with moyamoya disease. Poor filling of MCA branches was seen on bilateral ICA angiography. Left vertebral artery angiography ( $\mathbf{f}$ - anteroposterior, $\mathbf{g}$ - lateral) shows reconstitution of the majority of the MCA territories bilaterally via pial-collateral flow over the cerebral convexities from the distal posterior cerebral artery branches. 
vertebral angiography (fig. 1f, g) showed extensive pial-collateral flow over the cerebral convexities from the distal posterior cerebral artery branches with reconstitution of distal MCA territories bilaterally. The patient ultimately underwent bilateral pial synangiosis surgery 2 months later, with no subsequent neurological deficits. More surgically relevant aspects of this patient's treatment have been recently described elsewhere [2].

\section{Discussion}

$\mathrm{Hb}$ Casper (Southampton) is the substitution of leucine 106 (G8) of the $\beta$-chain by proline, characterized as a de novo mutation in a handful of patients [3-5]. It consists of anemia, notable hemolysis and splenomegaly, along with recurrent infections with hemolytic crises [6], which our patient previously experienced. Neurological symptoms have not been previously reported.

After a thorough investigation, we believe that the patient's strokes are due to moyamoya disease, which consists of large-vessel occlusions with extensive vascular collateralization. Given the lack of other causes of moyamoya disease, her hemoglobinopathy is felt to be the most likely etiology. Moyamoya has been extensively described in sickle cell disease [7]. Similar findings were noted in our patient, including leptomeningeal collaterals and increased vascular signal on FLAIR imaging (fig. 1c, arrow, 'ivy sign') [8]. Besides sickle cell disease in which occlusion is noted in large vessels possibly due to a proliferative vasculopathy and ultimately resulting in stenosis [9], evidence of moyamoya disease has been noted in $\beta$-thalassemia [10] and several other rare hemolytic anemias including Hb Fairfax [10] and Hb Alesha [11]. Whether transfusion therapy, as used in sickle cell disease, is indicated in patients with moyamoya disease due to other hemoglobinopathies is unknown [10]. If she develops new deficits, transfusion will be considered.

\section{Disclosure Statement}

The authors have no financial conflicts of interest.

\section{References}

1 Greenwald JG: Stroke, sickle cell trait, and oral contraceptives. Ann Intern Med 1970;72:960.

$\longrightarrow 2$ Delavari N, Strahle J, Maher CO: Moyomoya syndrome associated with hemoglobin Southampton (Casper). Pediatr Neurosurg 2014;49:307-310.

3 Koler RD, Jones RT, Bigley RH, Litt M, Lovrien E, Brooks R, et al: Hemoglobin Casper: beta 106(G8) Leu leads to Pro; a contemporary mutation. Am J Med 1973;55:549-558.

4 Hyde RD, Hall MD, Wiltshire BG, Lehmann H: Haemoglobin Southampton, 106 (G8) Leu leads to Pro: an unstable variant producing severe hemolysis. Lancet 1972;7788:1170-1172.

5 Wajcman H, Gacon G, Labie D, Koler RD, Jones RT: Isolation and functional characterization of hemoglobin Casper: beta106(G8) Leu replaced by Pro. Biochemistry 1975;14:5017-5020.

6 Heintz NH, Howard PL: Hemoglobin Southampton (Casper): characterization of the base mutation. Am J Hematol 1989;30:1-3.

7 Stockman J, Nigro M, Miskhkin M, Oski F: Occlusion of large cerebral vessels in sickle-cell anemia. N Engl J Med 1972;287:846-847.

8 Maeda M, Tsuchida C: 'Ivy sign' on fluid-attenuated inversion-recovery images in childhood moyamoya disease. AJNR Am J Neuroradiol 1999;20:1836-1838.

9 Lanzkowsky P: Hemoglobinopathies; in Manual of Pediatric Hematology and Oncology, ed 5. Amsterdam, Elsevier, 2011, pp 200-246.

10 Marden FA, Putman CM, Grant JM, Greenberg J: Moyamoya disease associated with hemoglobin Fairfax and beta-thalassemia. Pediatr Neurol 2008;38:130-132.

-11 Brockmann K, Stolpe S, Fels C, Khan N: Moyamoya syndrome associated with hemolytic anemia due to $\mathrm{Hb}$ Alesha. J Ped Hematol Oncol 2005;27:436-440. 